\title{
PAROXYSMAL TACHYCARDIA WITH EPISODIC UNCONSCIOUSNESS
}

\author{
BY \\ MARGARET HORAN and A. W. VENABLES \\ From the Royal Children's Hospital, Melbourne, Australia
}

(RECEIVED FOR PUBLICATION SEPTEMBER 5, 1961)

Unconsciousness due to disturbed cardiac output is seen most often as a result of acquired heart block with unstable ventricular rhythm. Less often it occurs as a result of an arrhythmia which may appear to be primary or else be associated with some obvious cardiac disease. Although unconsciousness due to this cause is relatively uncommon, it is important that the basic condition be recognized. Two such cases are reported and discussed with particular reference to the unusual arrhythmias recorded.

\section{Case Reports}

Case 1. Mary, a Maltese migrant girl, born on July 12, 1953, was seen first in February 1959, in a General Medical Out-patient Clinic at the Royal Children's Hospital, Melbourne, with a history of fainting turns for some 18 months. The turns occurred several times a week, always when she was frightened. She became pale and fell as though in a faint, recovering consciousness quite quickly and never twitching. Her pallor persisted for some time after recovery of consciousness.

Mary attended the Out-patient Department over the next 11 months. During this time she was treated with oral phenobarbitone, but continued to have attacks. Her mother observed that her heart beat rapidly during the attacks and took some time to return to a completely normal rate during the recovery phase.

Finally, in January 1960, she had an attack in the Outpatient Department. The pattern was as described. An electrocardiogram confirmed the presence of arrhythmia. This and subsequent recordings showed that the arrhythmia was of unusual type, varying in pattern (Fig. 1). At times it appeared to be basically supraventricular, although with variable complexes (Type 1). At other times there were periods of regularly alternating bi-directional complexes of uncertain origin (Type 2). In one attack there was ventricular fibrillation lasting for approximately 30 seconds (Type 3). This attack was the result of deliberately induced emotional upset, caused by the threat of making her walk downstairs, to which she had a particular aversion.

Between attacks she appeared well without any evidence of cardiac abnormality, and her electrocardiogram was normal (Fig. 2).
The attacks continued and at times were severe, with cyanosis and a deathlike appearance. Because of this and the consequent anxiety, she was admitted on two occasions for observation. Quinidine was given in progressively increasing dosage up to $360 \mathrm{mg}$. three times a day without producing control. At this stage her parents believed that she was being made worse by treatment. They said she was nauseated and, on the advice of their local practitioner, they discontinued quinidine.

At this time, April 1960, she ceased attending hospital, so that no other therapy could be tried. Her mother was then pregnant, and this appeared to aggravate the tension in the household.

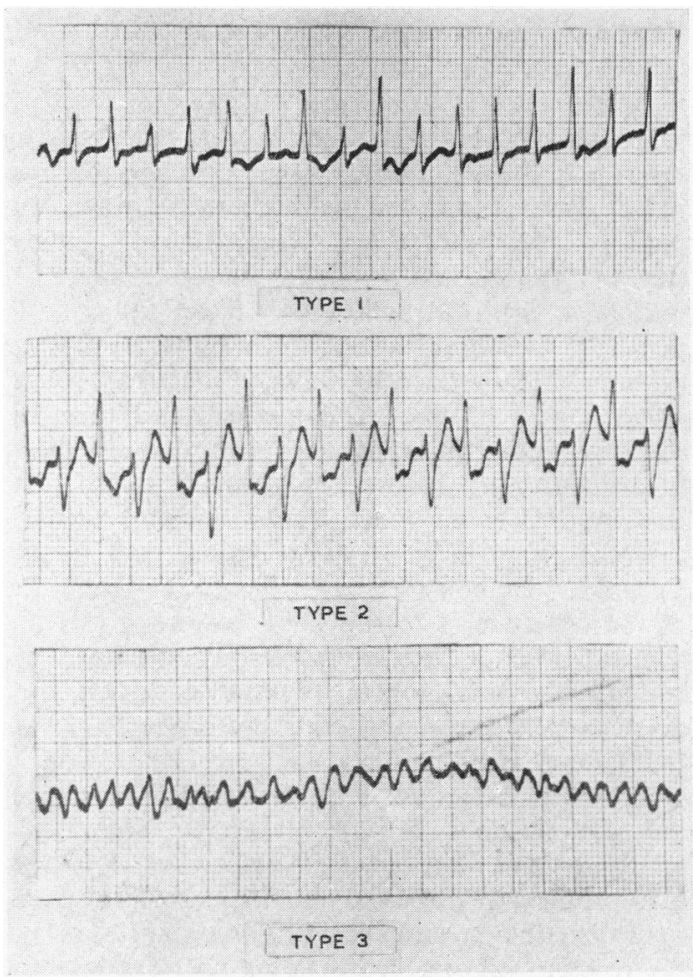

Fig. 1.-Arrhythmias present in Case 1. 


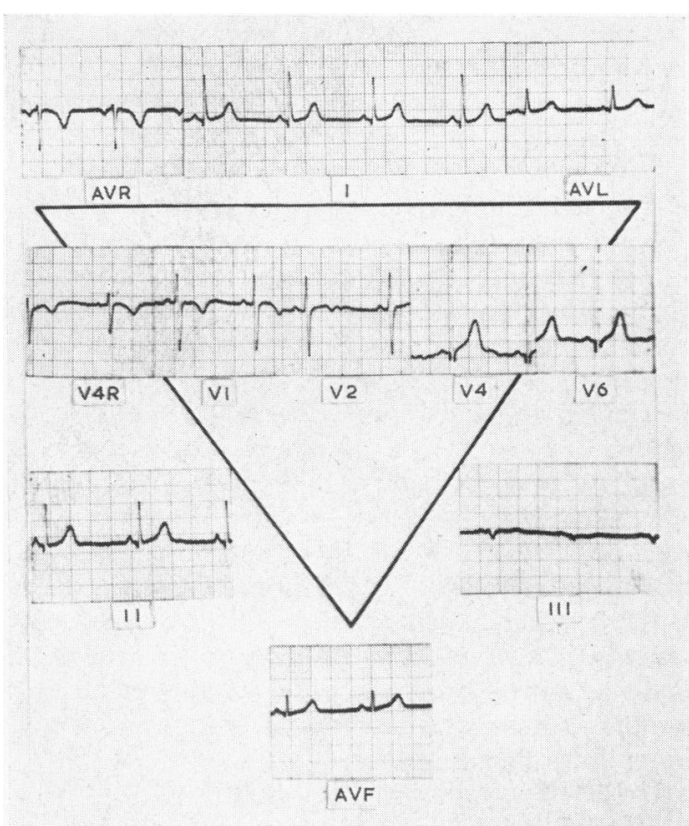

FIG. 2.-Normal electrocardiogram, Case 1.

During the next few months Mary attended a number of general practitioners and finally a herbalist. The herbalist diagnosed a 'nervous heart' and prescribed medication of unknown nature. Thereafter the attacks decreased considerably and virtually ceased. Late in 1960 the family was involved in a motor-car accident, the expenses of which forced them to cease taking Mary to the herbalist. After this Mary remained reasonably well, although with some return of less severe attacks. Both she and her parents became less apprehensive and Mary was able to attend school regularly.

In April 1961, she returned to the hospital for review in response to a letter regarding her progress. An electrocardiogram showed the development of abnormal beats and tachycardia after exertion. Brief periods of wandering atrial pacemaker were recorded during slowing of her heart produced by carotid sinus pressure. The previous, readily evoked emotional disturbance could not be reproduced.

Case 2. Peter, now aged 16 years, attended the Medical Out-patient Department of the Royal Children's Hospital from the age of 10 years. He had had fainting turns from the age of 3 years. These occurred about five or six times a year. He was stated to become pale, limp, then unconscious, and finally to twitch. The turns were interpreted as being due to epilepsy and he was treated with phenytoin. Electroencephalography was at that time not easily obtained and was not performed. At the age of $12 \frac{1}{2}$ years cardiological opinion was sought after successful resuscitation from cardiac arrest which occurred during mastoidectomy for chronic mastoiditis. At this time gross cardiomegaly was first recognized. Clinical examination suggested severe pulmonary hypertension. Subsequent investigation confirmed this without elucidating the aetiology.

From that time his course has been one of fairly steady deterioration with considerable disability associated with sub-acute right ventricular failure. A mitral systolic murmur suggests the possibility of left heart involvement, perhaps due to some form of myopathy.

Not long after the recognition of cardiac abnormality, attacks were observed in the hospital for the first time. It was clear that he had cardiac arrhythmia associated with pallor and a weak, irregular pulse. His parents confirmed the similarity of the observed attacks to those which they had previously described.

Electrocardiograms revealed the presence of atrial flutter with two to one block, the atrial flutter waves occurring at a rate of 300 per minute (Fig. 3). His

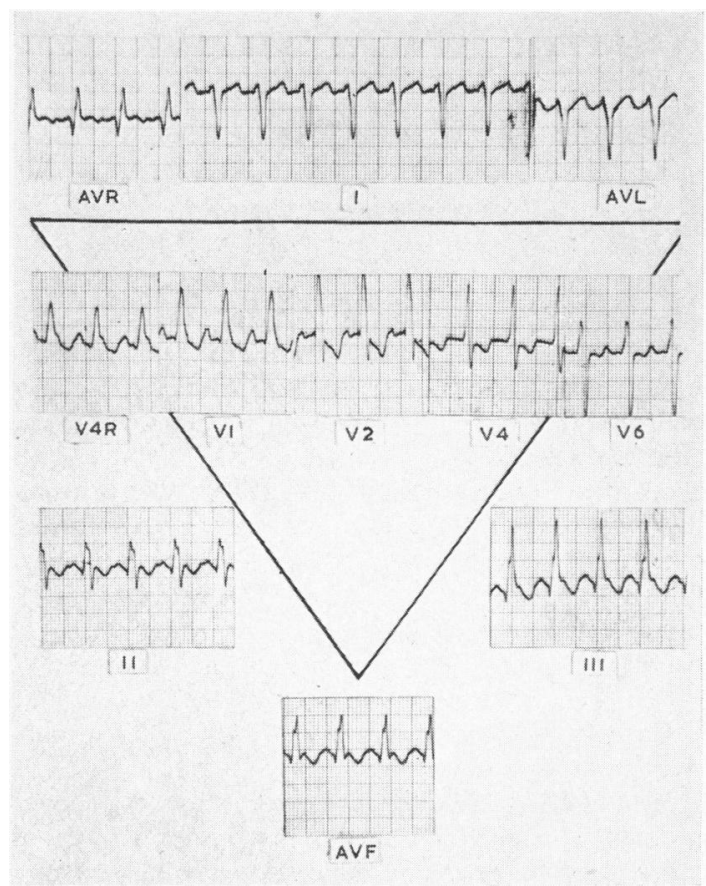

FIG. 3.-Arrhythmia, Case 2.

electrocardiogram between attacks showed right ventricular hypertrophy (Fig. 4). Digitalis, given because of heart failure, did not control the attacks satisfactorily. Quinidine, which had been given inadvertently before the true nature of the arrhythmia had been revealed by review of the available electrocardiograms, led to an increased severity of attacks. The bouts of unconsciousness became more prolonged and cardiac pain occurred. 


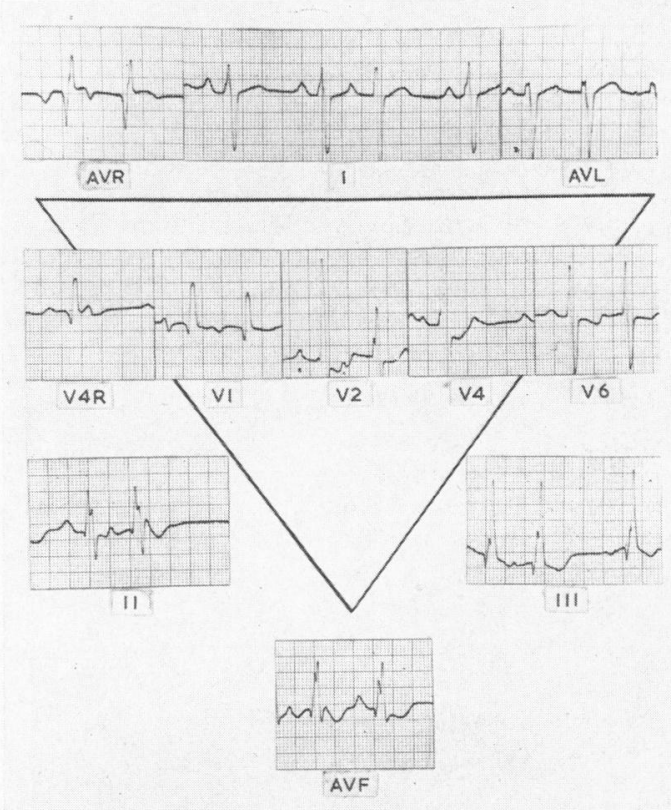

FIG. 4.-Electrocardiogram between attacks, Case 1.

\section{Discussion}

The arrhythmias most commonly seen in childhood are paroxysmal tachycardias of supraventricular origin. These usually have a characteristic sudden onset and offset. There may be no symptoms other than palpitation. Occasionally dizziness occurs, but this is unusual. In infancy even fairly short attacks often precipitate heart failure. Attacks of paroxysmal tachycardia may be precipitated or potentiated by emotional upset but usually occur without such a relation.

Case 1. This case exhibits a number of features worthy of discussion.

In this child attacks were usually associated with periods of emotional upset, due either to fear or more often to what one might describe as tantrums. There was also evidence of a relation between the attacks and exertion.

Although the electrocardiographic evidence excluded an abrupt change in rhythm, clinically she exhibited a fairly sudden change in consciousness associated with tachycardia. Cardiac output was clearly greatly reduced as the cerebral effects were profound, and on a number of occasions the child appeared on superficial inspection to have died. The rates and rhythms recorded were in keeping with this effect on output. Recovery, unlike the common arrhythmias referred to, was gradual with persistent pallor and progressive slowing of the heart rate as observed by the mother and documented ultimately by electrocardiography.

A diagnosis of epilepsy was originally considered in this child, although the episodes were atypical. The mother's observation of a disordered heart beat gave the clue to the real nature of the condition. The gradual recovery from the attacks was fully explained once adequate electrocardiographic documentation was obtained. The gradual recovery, the pallor, and the mode of onset were all evidence against epilepsy which was only suggested by the episodic unconsciousness. A series of simple breath-holding attacks might have been considered as a possible cause for this condition, as breathholding attacks occur under circumstances similar to those which precipitated this child's turns. The absence of observed breath-holding, and the pallor as distinct from cyanosis, excluded this much more common cause of unconsciousness accompanying crying with fear or anger.

These observations stress the need for taking a detailed history and for adequate investigation in any case of episodic unconsciousness with unusual features.

The course of the illness, as yet not final, is of interest. The period of maximum disability was associated with considerable anxiety in the parents, largely related to the child herself, but aggravated, as far as could be assessed, by the mother's pregnancy. Detailed assessment of the situation was hindered by language difficulties. A further factor, however, appeared to be fear and distrust of therapy on the part of the parents. The considerable improvement attributed to the herbalist's unknown medication coincided with the successful outcome of the pregnancy. Mild deterioration occurred following the motor-car accident and the subsequent financial problems. This deterioration was attributed by the family to cessation of the herbalist's medication. At that time Mary's emotional adjustment appeared much better than earlier. Whether this was the cause or the result of her improvement is not known.

Although she has improved, there is still a tendency to arrhythmia. This is disclosed by the reaction to exertion. The types of arrhythmia recorded earlier, particularly the episode of ventricular fibrillation, must make prognosis uncertain despite the lack of evidence of cardiac abnormality. Clinical examination, radiographs and resting electrocardiograms are all normal. Periodic followup should now be possible with the re-establishment of contact with the family. 
The actual arrhythmias recorded are quite unusual. The first, most common pattern, which appears to provide the mode of onset or initial disturbance, has some resemblance to the pattern of chaotic heart action described by Katz and Pick (1956). This rhythm at times seems to comprise sinus beats interspersed with multiple ventricular extrasystoles. At other times the pattern is less clear, with regular beats of multiple type, less readily assessed, but probably resulting from variable conduction disturbance.

The pattern of alternating bi-directional complexes recorded during attacks is also rare. Such arrhythmias are usually due to digitalis intoxication (Katz and Pick, 1956), although other examples have been recorded (Pick and Langendorf, 1960). In the tracings from this case, no $\mathbf{P}$ waves can be demonstrated during the bi-directional tachycardia. Both the cycle length and the width of the ventricular complexes alternate slightly. The wider ventricular complex follows the shorter cycle. In one strip the cycle lengths were 0.22 second and 0.26 second, the respective QRS widths being $0 \cdot 12$ second and 0.08 second. Alternating bundle branch block superimposed on a supraventricular tachycardia is possible, although the varying cycle length requires a more complicated explanation. There is, however, no clear evidence of a ventricular origin for this rhythm, except perhaps the association with one recorded attack of ventricular fibrillation. Some short bursts of alternation recorded are obviously due to alternating ventricular extrasystoles. The occurrence of a dual rhythm with supraventricular and ventricular foci seems improbable in spite of this observation.

Ventricular fibrillation of paroxysmal nature unassociated with cardiac abnormality is also extremely rare. In this girl its occurrence in an attack deliberately evoked under observation suggests its possible occurrence in other severe attacks not so documented. It provides a very adequate explanation for the severity of her symptoms and one of the main grounds for doubt in prognosis. This attack lasted approximately 30 seconds, after which the patient was pale, cyanosed and frothing at the mouth, although not actually fitting. Electrocardiographically, this episode ended with a fairly sudden change to less characteristic arrhythmia and ultimate return by the usual chaotic pattern to sinus rhythm.

One can only speculate regarding the mode of precipitation of these attacks. The circumstances suggest excessive liberation of adrenalin combined with a particularly sensitive myocardium. There is no evidence pointing to phaeochromocytoma either in the observations or in the history. Presentation of phaeochromocytoma with paroxysmal tachycardia has been described (Watt, Bush and Walker, 1960). The effect of phentolamine was not tried owing to the lapse in attendance while attempts were being made to assess the value of quinidine alone in full dosage. Quinidine was used because of the possible ventricular nature of much of the arrhythmia, and because of the improbability of decreasing the factor of anxiety even by psychotherapy, particularly in view of the problems of language, the mother's pregnancy, and her fear of the child's sudden death. Evidence of quinidine toxicity amounted to nausea only at a dose of $1,080 \mathrm{mg}$. per day (approximately $60 \mathrm{mg}$. per $\mathrm{kg}$.). The blood level of quinidine could not be estimated at that time. The effect of exogenous adrenalin was not tried because of the potential risk if, as seemed likely, adrenalin did precipitate the attacks.

Case 2. In this patient, the arrhythmia, although still of somewhat uncommon type for the age group, was associated with gross cardiac abnormality. The basis of this lesion is incompletely elucidated as yet. Signs of cardiac disorder were overlooked, and the real cause of his attacks of unconsciousness was missed until the time of his cardiac arrest.

The second case is reported briefly to reinforce the lesson of the first. The types of arrhythmia present in these cases explain the severity of their cerebral symptoms.

\section{Summary}

Case details of two patients are given to demonstrate the possible relation of paroxysmal tachycardia to episodic loss of consciousness in children.

Emotional upset and effort precipitated the unusual rhythms of chaotic heart action, bi-directional tachycardia, and ventricular fibrillation in Case 1, a girl, in whom there was no other evidence of cardiovascular abnormality.

Pulmonary hypertension of undetermined aetiology was present in Case 2. The abnormal rhythm in this patient was atrial flutter with, ordinarily, two to one block.

\section{REFERENCES}

Katz, L. N. and Pick, A. (1956). Clinical Electrocardiography. Part I: The Arrhythmias, with an Atlas of Electrocardiograms. Kimpton, London.

Pick, A. and Langendorf, R. (1960). Differentiation of supraventricular and ventricular tachycardias. Progr. cardiovasc. Dis. 2, 391.

Watt, J.' M., Bush, R. T. and Walker, L. J. (1960). Phaeochromocytoma presenting as paroxysmal tachycardia. N.Z. med. J., 59,541 . 